\title{
Preface: Invited Issue Editor, Professor James T. Rutka, and the Pediatric Malignant Brain Cancers in the Molecular Era
}

\author{
Seung-Ki Kim, M.D., Ph.D., ${ }^{1,2}$ Kyu-Chang Wang, M.D., Ph.D.' \\ Editor of the 'Pediatric Issue,", Journal of Korean Neurosurgical Society, Seoul Korea \\ Chair, Publication Committee, 2 The Korean Society for Pediatric Neurosurgery, Seoul, Korea
}

Since the Korean Journal of Pediatric Neurosurgery $(K J P N)$ was first issued by the Korean Society for Pediatric Neurosurgery (KSPN) in 2004, KJPN has made efforts to ensure that clinical experiences are shared with updated knowledge and to provide education on pediatric neurosurgery topics. However, as a domestic journal, at times KJPN had difficulty in publishing high impact papers on this topic. Therefore, KSPN decided to discontinue the publication of KJPN and instead to publish an official journal the Pediatric Issue as a supplement to the Journal of Korean Neurosurgical Society (JKNS) in 2014. The Pediatric Issue aims to share updated information on specific topics of pediatric neurosurgery with general neurosurgeons and also those in other subspecialties.

Ever since, JKNS : Pediatric Issue has been published annually from 2015. The topics of the Pediatric Issue so far have been Moyamoya disease (edited by Professor Seung-Ki Kim), Craniosynostosis (invited editor, Professor Joon-Ki Kang) and Neuroendoscopy (invited editor, Professor JoongUhn Choi).

The topic of the Pediatric Issue in 2018 is 'Pediatric malignant brain cancers in the molecular era' and was organized

- Received : December 11, 2017 •Revised : January 3, 2018 •Accepted : January 9, 2018

- Address for reprints : Kyu-Chang Wang, M.D., Ph.D.

Division of Pediatric Neurosurgery, Seoul National University Children's Hospital, Seoul National University College of Medicine, 101 Daehak-ro, Jongno-gu, Seoul 03080, Korea

Tel : +82-2-2072-3489, Fax : +82-2-2072-0274, E-mail : kcwang@snu.ac.kr by the invited editor, Professor James T. Rutka, a world-renowned leader of pediatric neuro-oncology (Fig. 1).

Professor James T. Rutka, born in Toronto, was educated at Princeton University (1975-1977), and Queen's University Medical School (1977-1981). Dr. Rutka served his internship at McGill University (1981-1982) before entering the University of Toronto Neurosurgery Training Program in 1982. His training included a research fellowship at the Brain Tumor Research Centre, the University of California San Francisco

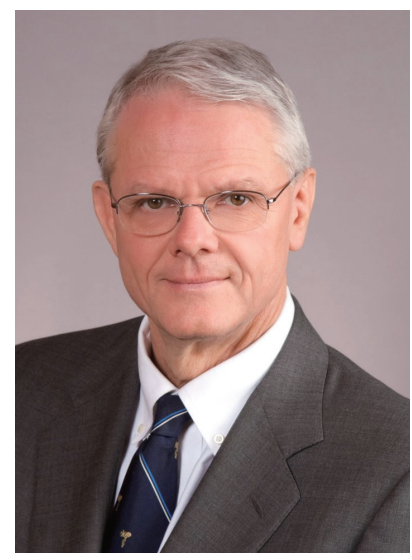

Fig. 1. Portrait of Professor James T. Rutka.

This is an Open Access article distributed under the terms of the Creative Commons Attribution Non-Commercial License (http://creativecommons.org/licenses/by-nc/4.0) which permits unrestricted non-commercial use, distribution, and reproduction in any medium, provided the original work is properly cited. 
where he obtained his Ph.D. in Experimental Pathology (1984-1987).

Dr. Rutka assumed his appointment in the Division of Neurosurgery, the Department of Surgery, the University of Toronto in 1990. He is a pediatric neurosurgeon whose clinical practice has been at the Hospital for Sick Children. Dr. Rutka's primary research and clinical interests are related to the science and surgery of human brain tumors and epilepsy. He has achieved over 500 peer reviewed publications.

In 1999, Dr. Rutka was promoted to Professor in the Department of Surgery at the University of Toronto, and was appointed to the Dan Family Chair in the Division of Neurosurgery-a position he held until 2011. In 2009, he became the Honored Guest at the Congress of Neurological Surgeons' Annual Meeting. In 2010-2011, Dr. Rutka served as President of the American Association of Neurological Surgeons. In 2011, he became President of the World Academy of Neurological Surgery, President of the American Academy of Neurological Surgery, and Fellow of the Royal Society of Canada. In the same year, he was appointed as the RS McLaughlin Professor and Chair of the Department of Surgery at the University of Toronto. In 2013, he became the first Canadian to be appointed as Editor-in-Chief of the Journal of Neurosurgery, and was inducted as a Member of

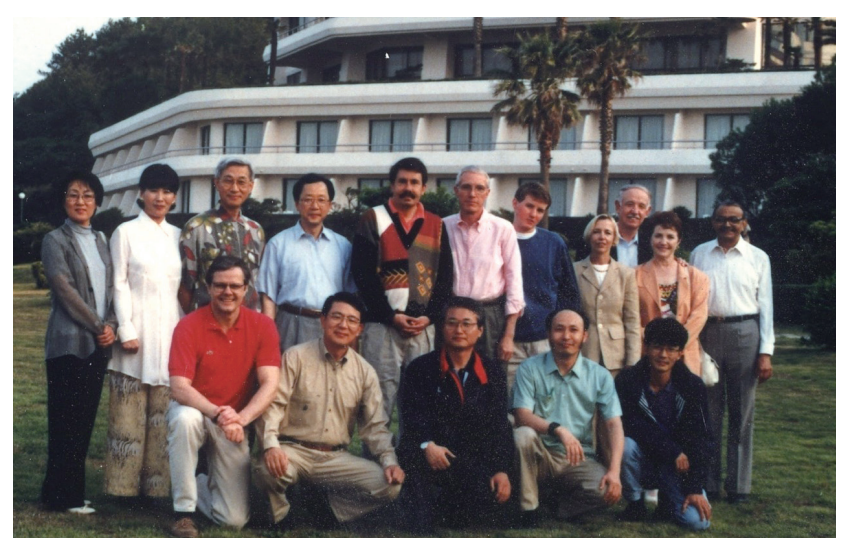

Fig. 2. James T. Rutka in Jeju, 1997 after the International Symposium on the 10th Anniversary of Korean Society for Pediatric Neurosurgery. World famous pediatric neurosurgeons joined the symposium and delivered inspiring lectures. After the symposium, invited speakers had a tour in Jeju (counter-clockwise from bottom left : James T. Rutka, Joong-Uhn Choi, Shi Hun Song, Kyu-Chang Wang, Seung-Ki Kim, Sanat N. Bhgwati, David G. McLone and his wife, Gordon McComb and his wife and son, Leslie N. Sutton, Byung-Kyu Cho, Yoon Sun Hahn and his wife, and wife of JoongUhn Choi). the Order of Ontario. In 2015, he received the Margolese National Brain Disorders Prize from the University of British Columbia, the Robert L. Noble Award from the Canadian Cancer Society, and was made an Officer of the Order of Canada. In 2017, he received an honorary degree, Doctor of Science, from his alma mater, Queen's University where he went to medical school. Dr. Rutka is married to Mari Rutka and they have three children together: Daniel, Hana and Marissa.

Because of his achievements in Pediatric Neurosurgery, Dr. Rutka was invited as the editor of Pediatric Issue of 2018 : Pediatric malignant brain cancers in the molecular era. He has maintained a strong tie with the Korean Neurosurgical Society since 1997 when he was invited as a speaker to the International Symposium on the 10th Anniversary of KSPN (Fig. 2). At that symposium he delivered three fascinating lectures titled 'Update in neuro-oncological basic research', 'Frameless stereotactic surgery in pediatric neurosurgery', and 'Brain stem tumor'. So far many Korean neurosurgeons have had a privilege of receiving training experience at Dr. Rutka's lab and clinic.

In 2016, the World Health Organization Classification of Tumors of the Central Nervous System underwent major revision with molecular profiling of brain tumours receiving stronger emphasis. Accordingly, integrated diagnosis combining histology and molecular characteristics has been widely recommended for brain tumors, which amounts to a major paradigm shift. Now, further refinement of risk stratification is needed in order to improve patient prognosis, and to constitute the basis for finding therapeutic targets within each tumor entity.

Dr. Rutka is pleased to share these advancements and trends with all members of Korean Neurosurgical Society. Twelve articles were contributed by leading researchers in this field in Korea and Canada; in particular, his colleagues from the Toronto group have actively participated in the major changes that have occurred in our understanding of pediatric malignant brain cancers in the molecular era : Dr. Michael Taylor has written on medulloblastoma, Dr. Annie Huang on atypical teratoid/rhabdoid tumor, Dr. James T. Rutka on diffuse midline glioma, H3 K27M-mutant, and Dr. Uri Tabori on high grade glioma and its genetic basis. Finally, Dr. Rutka has concluded this special issue by sharing his views on the current practice of pediatric neuro-on- 
cology, and its many exciting future perspectives.

We appreciate the contributions made by all the participating experts, especially those from the University of Toronto, for their active participation as well as Professor Rut$\mathrm{ka}$ for his enthusiasm as the invited editor.

\section{CONFLICTS OF INTEREST}

No potential conflict of interest relevant to this article was reported.

\section{INFORMED CONSENT}

This type of study does not require informed consent.

\section{- Acknowledgements}

We deeply appreciate Ms. Bo-Young Lee for the English editing of this article. 